\title{
Bioluminescent Imaging of Pneumococcal Otitis Media in Chinchillas
}

\author{
A THESIS \\ SUBMITTED TO THE FACULTY OF \\ UNIVERSITY OF MINNESOTA \\ BY
}

\author{
Alan Wellington Johnson, MD
IN PARTIAL FULFILLMENT OF THE REQUIREMENTS FOR THE DEGREE OF \\ MASTER OF SCIENCE \\ Co-Advisor: Jizhen Lin, MD \\ Co-Advisor: James Sidman, MD
}

June 2013 
This thesis also published as:

Alan W Johnson; James D Sidman; Jizhen Lin. Bioluminescent Imaging of Pneumococcal Otitis Media in Chinchillas. Annals of Otology, Rhinology \& Laryngology. 122(5):344-352.

Permission granted for publication by Annals Publishing Company.

(C) 2013 Annals Publishing Company. All rights reserved. 


\section{Acknowledgements}

With special thanks to Dr. James Sidman, Dr. Jizhen Lin, Dr. Angela Black, Dr. Frank Ondrey, Beverly Wuertz, and the Otolaryngology - Head \& Neck Surgery Department at the University of Minnesota. 


\section{Dedication}

This thesis is dedicated to:

Dr. Samuel Levine and Dr. Deborah Kacmarynski - my initial inspiration to combine my engineering and otolaryngology interests,

and to:

Katy, Elliot, and Evan - my daily inspiration. 


\begin{abstract}
Objectives: Bioluminescent imaging has emerged as a powerful tool for monitoring the pathological process of infections in animals. The purpose of this study was to harness this new tool for objective assessment of acute otitis media (AOM) in animals with and without antibiotic interventions. Methods: Thirty-six healthy chinchillas, free of middle ear infections, were randomly divided into a control group and a group that received amoxicillin treatment. Bioluminescent Streptococcus pneumoniae (Xen 10) was injected into the epitympanic bullae of chinchillas (50 colony-forming units each) for induction of AOM. The infectious process of Xen 10 in the bullae of living animals with and without antibiotic interventions was monitored in real time with bioluminescence equipment.

Results: A dynamic change of bioluminescent signals in the bullae of chinchillas from days 1 to 14 was observed after Xen 10 injection. Amoxicillin treatment reduced the bioluminescent signals in the bullae of chinchillas compared with controls. The AOM persisted for 14 days, and middle ear effusion for 6 weeks, in the control animals, whereas AOM lasted for 2 days, and effusion for 6 to 12 days, in the antibiotic-treated animals. Conclusions: Bioluminescent imaging provides an innovative method for assessment of the bacterial loads in the middle ear of chinchillas in a real-time manner and is very useful for objective evaluation of the efficacy of therapeutic interventions.
\end{abstract}




\section{Table of Contents}

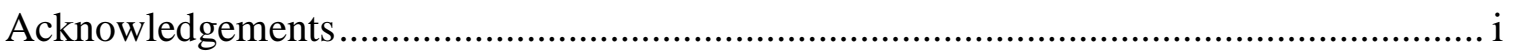

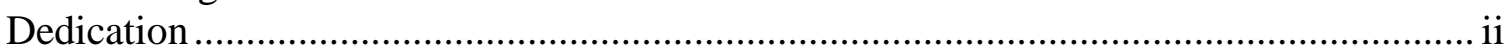

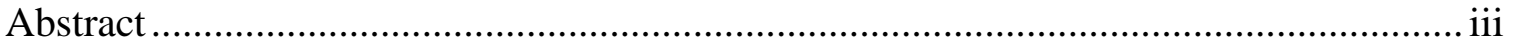

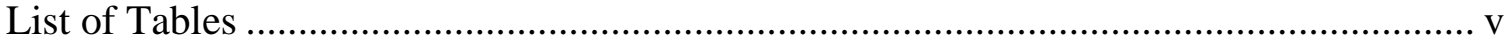

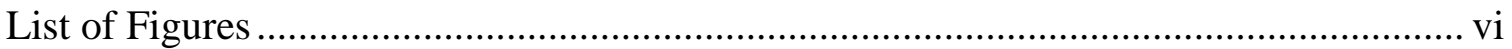

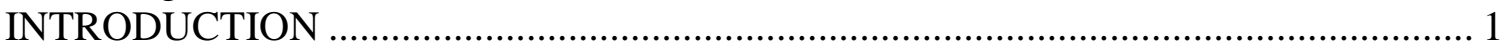

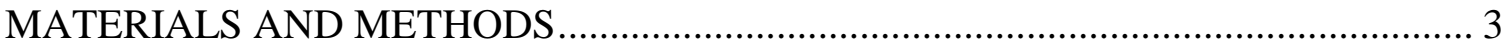

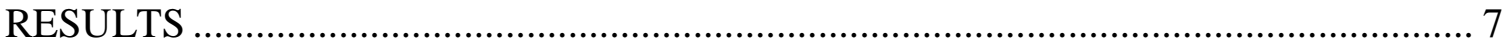

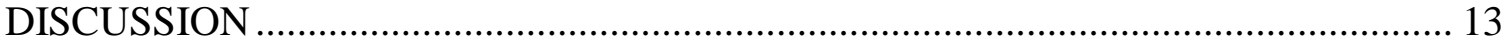

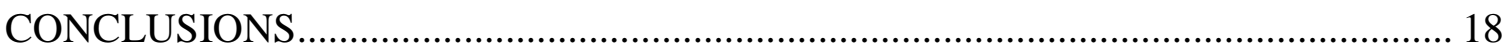

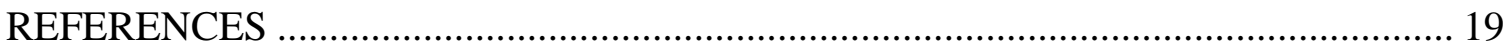




\section{List of Tables}

Table 1:

Outcomes following injection of bioluminescent Streptococcus pneumonia in chinchillas

with and without Amoxicillin treatment......................................... 


\section{List of Figures}

Figure 1:

View of top of chinchilla head with animal in prone position $\ldots \ldots \ldots \ldots \ldots \ldots \ldots \ldots \ldots \ldots$

Figure 2:

Time-dependent progression of acute otitis media in parallel with intensity changes of

bioluminescent signals.................................................. 11

Figure 3:

Dissemination of acute otitis media from one bulla to other of chinchilla with time lag..12 


\section{INTRODUCTION}

Otitis media is one of the most common infectious diseases in children, accounting for 22 million visits to physicians per year in the United States. ${ }^{1}$ By 1 year of age, most children have experienced an episode of acute otitis media (AOM), and by 3 years of age, more than $80 \%$ have had at least 1 episode. ${ }^{2}$ The annual cost in the United States for its treatment is estimated to be on the order of $\$ 5$ billion. ${ }^{3}$ Despite aggressive medical treatment, otitis media remains a common cause of acquired hearing loss. ${ }^{4}$ Antibiotic treatment of otitis media has become less effective in recent years with the alarming emergence of multidrug-resistant organisms ${ }^{5,6}$ and has only a minimal effect on the longterm resolution of middle ear effusion. ${ }^{7,8}$ For us to better understand the infectious process of middle ear pathogens and to evaluate the efficacy of antibiotics, animal models are critical.

Through the years, animal models have been developed for AOM studies. ${ }^{9-11}$ Because of anatomic limitations, none of the models allow direct visualization of the actual infection process of AOM in the middle ear cavity of living animals. The occurrence of infection is assumed from the pathological changes of animals' middle ear mucosa, but is not evidenced by the bacterial growth of pathogens in the middle ear of animals in a timedependent manner. Under certain circumstances, dead bacteria or their metabolites such as cell wall envelope components or DNA are sufficient to induce an inflammatory response of the middle ear mucosa. ${ }^{12,13}$ Unfortunately, these mucosal responses are counted as middle ear infections even though there are no actual middle ear infections. 
There is a need to establish a new method to distinguish genuine middle ear infections from middle ear inflammatory responses that are not induced by living microorganisms. From the histologic perspective, it is difficult to do so, because the inflammatory response induced by nonliving pathogens is not as different from a response to infection as one would think. In the past, invasive methods, including trans-tympanic needle aspiration and animal sacrifice, were typically used to determine the presence of infection. Although animal models allow for microscopic inspection of the tympanic membrane, the degree of infection is still a subjective estimate. Microscopic inspection cannot reliably distinguish AOM (active infection) from otitis media with effusion (the fluid retained after infection). Trans-tympanic aspiration can spare the need to euthanize animals, but the perforation left in the tympanic membrane invalidates future data by altering the disease course. Further, although sequentially euthanized animals can provide insights into the temporal course of disease, the middle ear inflammatory responses vary from animal to animal, mandating that high numbers of animals be euthanized to obtain statistically relevant data.

To establish an animal model that can differentiate the sources of inflammation, bioluminescent bacteria might allow scientists to directly visualize the growth of bacteria without resorting to invasive measures such as trans-tympanic needle aspiration or animal sacrifice. Bioluminescent imaging provides us with a powerful new tool by offering noninvasive monitoring of real-time infection. Multiple reviews have emphasized the 
strength of this technology. ${ }^{11,14,15}$ We hypothesized that early detection and real-time monitoring of AOM would be possible with bioluminescent pneumococcus in the chinchilla model of otitis media. Further, we expected that bioluminescent monitoring would enhance our ability to detect subtleties of the disease that were not possible to detect with traditional methods.

\section{MATERIALS AND METHODS}

Bioluminescent Bacterial Strain. Bioluminescent bacteria were obtained from Caliper Life Sciences (Hopkinton, Massachusetts), formerly Xenogen, in the form of Xen 10 (Streptococcus pneumoniae, serotype 3). The development of this bioluminescent strain of pneumococcus was described by Francis et al. ${ }^{16}$ Serotype 3 is known to be a middle ear pathogen. It was shown in a multinational review to be a leading serotype causing otitis media; it is not included in the 7 -valent pneumococcus vaccine, ${ }^{17}$ but is included in the 23-valent pneumococcal vaccine. For bioluminescence, this bacterium features integration of the Photorhabdus luminescens lux operon into its bacterial genome. The bacterium does not require the addition of an exogenous substrate (i.e., luciferin) to exhibit luminescence. Additionally, the bacteria possess kanamycin resistance, which helps to minimize contamination during microbiological manipulation.

Upon receiving the bacteria, we cultured them at $37^{\circ} \mathrm{C}$ with $5 \%$ carbon dioxide in brainheart infusion with $5 \%$ fetal bovine serum (BHI-FBS) culture medium and found them to be bioluminescent in the IVIS 50 imaging system (Caliper Life Sciences). The bacteria 
were subsequently frozen in a glycerol suspension at $-80^{\circ} \mathrm{C}$. In subsequent experiments, we used this frozen stock to maintain standardization across experiments.

Chinchilla Model of AOM. Thirty-six adult chinchillas (Chinchilla laniger), weight 400 to $700 \mathrm{~g}$, were obtained from Moulton Chinchilla Ranch (Rochester, Minnesota). The chinchillas were inspected for premorbid illnesses by Research Animal Resources veterinarians at the University of Minnesota. The animals were also evaluated for premorbid ear disease by otomicroscopy and tympanometry. Those demonstrating illness were excluded. Thirty-six animals were used in this study. After exclusion, 33 animals were divided into 3 groups for study of the infectious process of Xen 10 in chinchillas. Group 1 had 12 animals with inoculum of 800 colony-forming units (CFU) per bulla. Groups 2 and 3 had 10 and 11 animals with inocula of 50 CFU per bulla (see Table for details). In all animals, the left middle ear-bulla was inoculated and the right side was kept as a control. The animals were allowed to acclimatize to the animal housing facilities for 1 week before inoculation. All animal handling and infectious agent protocols were approved by the University of Minnesota's Institutional Animal Care and Use Committee and the Institutional Biosafety Committee, respectively.

To inoculate the animals, we re-cultured bacteria from frozen stock at $37^{\circ} \mathrm{C}$ with $5 \%$ carbon dioxide in BHI-FBS and grew them to a concentration between $10^{8}$ and $10^{9}$ CFU/mL. Optical density correlations made with a Beckman DU 640 spectrophotometer 
(wavelength $600 \mathrm{~nm}$; Beckman Coulter, Brea, California) were used to confirm bacterial concentrations in this range. The cultures were then serially diluted in phosphate-buffered saline solution by a factor of $10^{5}$ or $10^{6}$. The animals were anesthetized with intramuscular injections of ketamine hydrochloride (20 to $25 \mathrm{mg} / \mathrm{kg}$ ). While the animals were under anesthesia and before injection, we removed the fur from the top of the head to visibly define the boundaries of the epitympanic bulla for accurate injection and for future bioluminescent imaging. Tuberculin syringes were used to deliver $0.1 \mathrm{~mL}$ of the bacterial solution into the epitympanic bulla of the chinchilla. During inoculation, bacteria were plated from the serially diluted mixture for definitive assessment of inoculum bacterial concentrations. This technique reliably delivered an average of 50 CFU per inoculation.

Antibiotic Therapy. Commercial amoxicillin suspension (400 mg/5 mL) was used to treat the animals in the antibiotic arm of the study. The animals were given the suspension twice a day by oral gavage for a 10-day course. This regimen is roughly equivalent to a human "low-dose" regimen of $30 \mathrm{mg} / \mathrm{kg}$ per day. Higher doses were not entertained, as chinchillas are susceptible to gastrointestinal bacterial eradication with amoxicillin. To prevent severe dehydration secondary to antibiotic-related diarrhea, we gave the animals receiving amoxicillin $0.5 \mathrm{mg}$ of Bene-Bac (PetAg, Inc., Hampshire, Illinois) probiotic gel daily via oral gavage. 
Semi-quantitative Bioluminescent Imaging. The IVIS 50 imaging system with Living Image software (Caliper Life Sciences) was used for all animal imaging studies. To establish the animals' premorbid state, we performed imaging studies before inoculation and immediately after inoculation to ascertain that no bioluminescent signal existed before infection. We performed imaging studies again at approximately 24 hours to establish that acute bacterial infection had occurred and that bacterial growth had reached the threshold of detection by the IVIS imager (more than $10^{4} \mathrm{CFU}$ ). Repeat imaging was performed every 2 to 3 days thereafter. The animals were anesthetized with intramuscular ketamine hydrochloride (20 to $25 \mathrm{mg} / \mathrm{kg}$ ) so that they would remain motionless during each 180-second imaging procedure. A color-gradient photo overlay represented the photon density from the bioluminescent bacterial infection. Quantitative assessments of total photon emissions were obtained with the Living Image software's region-of-interest function.

Otomicroscopy and Tympanometry. Concurrent with the bioluminescent imaging, we used otomicroscopy and tympanometry to compare the bioluminescent imaging to traditional methods of noninvasive assessment. A Wild Heerbrugg M650 surgical microscope (Heerbrugg, Switzerland) coupled with a Canon SureShot A520 digital camera to the side arm allowed for photography of the tympanic membrane. A GrasonStadler GSI 27A Autotymp tympanometer (Cardinal Health, Dublin, Ohio) produced tympanograms for assessment of tympanic membrane compliance. 


\section{RESULTS}

General Profile of Bioluminescent Otitis Media in Chinchillas. Chinchillas showed reliable infections by 24 to 48 hours when they were inoculated with the bioluminescent S pneumoniae (Xen 10) at a dose of 50 CFU per bulla. Peak bioluminescent intensity was detected in inoculated ears on day 3 for most animals. Some animals experienced similar levels of infection but developed labyrinthitis at approximately 48 hours. In the pilot study, a cohort of 12 animals that were inoculated with $800 \mathrm{CFU}$ per bulla, instead of 50 CFU per bulla, progressed to labyrinthitis within 36 to 48 hours. Three of these animals were started on oral amoxicillin (15 mg/kg dosed twice per day), which resolved their labyrinthitis. Those that did not receive amoxicillin all progressed to labyrinthitis and meningitis. The animals that developed meningitis were euthanized. Only 1 of the 9 animals that did not receive amoxicillin survived the infection. Of the 10 animals that received the $50 \mathrm{CFU}$ inoculations but no amoxicillin, 3 survived. All 11 of those that received amoxicillin within 24 to 48 hours of inoculation survived. Tympanometry and microscopy were performed during the experiment. The findings are summarized in the Table. 


\begin{tabular}{|c|c|c|c|c|c|c|c|}
\hline $\begin{array}{c}\text { No. of } \\
\text { Animals }\end{array}$ & $\begin{array}{l}\text { Dose } \\
(C F U)\end{array}$ & $\begin{array}{l}\text { Amoxicillin } \\
\text { Treatment }^{b}\end{array}$ & $\begin{array}{l}\text { No. That } \\
\text { Survived }\end{array}$ & Tympanometryc & Otoscopy $y^{d}$ & $\begin{array}{l}\text { Duration } \\
\text { of Effusion }\end{array}$ & Timeline \\
\hline 10 & 50 & No & 3 & Abnormal & Abnormal & $4-6 \mathrm{wk}$ & $6 \mathrm{wk}$ \\
\hline 11 & 50 & Yes & 11 & Abnormal & Abnormal & $6-12 \mathrm{~d}$ & $3 \mathrm{wk}$ \\
\hline \multirow[t]{2}{*}{$12^{e}$} & 800 & No 9 & 1 & $\mathrm{~N} / \mathrm{A}$ & N/A & $\mathrm{N} / \mathrm{A}$ & $<1$ wk \\
\hline & & Yes 3 & 3 & Abnormal & Abnormal & $6 \mathrm{wk}$ & $6 \mathrm{wk}$ \\
\hline \multicolumn{8}{|c|}{ CFU - colony-forming units; N/A - information not available. } \\
\hline \multicolumn{8}{|c|}{ aAnimals used in study after exclusion of preexisting conditions. } \\
\hline \multicolumn{8}{|c|}{ bAmoxicillin was given 24 to 48 hours after inoculation of Xen 10 . } \\
\hline \multicolumn{8}{|c|}{ cAbnormal - flat, type B profile consistent with fluid or infection behind tympanic membrane. } \\
\hline \multicolumn{8}{|c|}{ dAbnormal - erythematous tympanic membrane with purulent middle ear fluid. } \\
\hline \multicolumn{8}{|c|}{ 'Eight animals with dose of $800 \mathrm{CFU}$ were euthanized 2 to 3 days after Xen 10 inoculation. } \\
\hline
\end{tabular}

Detection of Bacterial Colonization in Bullae of Chinchillas. Figure 1A shows that

bioluminescent signals were not detected in the bulla of a chinchilla immediately after the injection, when injected bacteria had not yet colonized the middle ear, whereas

bioluminescent signals were detected in the bulla of the same chinchilla at 24 hours after the injection, when the injected bacteria had become established and formed colonies (Fig 1B). We noted that the signals confined to a limited area of the bulla at 24 hours after injection in animals inoculated with a dose of $50 \mathrm{CFU} / \mathrm{mL}$ per bulla were reliably shown to indicate AOM by 24 to 48 hours. There were individual differences between animals in terms of the occurrence and duration of AOM.

Effects of Animal Position on Detection of Bioluminescent Signals. To study whether bioluminescent signal intensity is related to animal position, we presented various angles (prone, supine, and lateral) of the bulla of a chinchilla to the IVIS 50 camera. We found that the bioluminescent signal intensity was relatively strong when the injected bulla was oriented toward the camera, that is, with the animal in the prone position (Fig 1C).

Obviously, the prone position orients the animal in relation to the camera so that there is 
minimal fur or tissue for the photons to traverse. The bioluminescent signal intensity was reduced when the injected bulla was oriented with the animal in the left lateral position (Fig 1D). In this position, bioluminescent signals are blocked by the tissue, skin, and fur. The bioluminescent signal intensity was relatively weak when the injected bulla was not oriented toward the camera (Fig 1E). In this position, bioluminescent signals are blocked not only by the tissue, skin, and fur on the right lateral side, but also by the midline tissue and bones. The bioluminescent signals were not detected when the injected bulla was oriented away from the camera with the animal in a supine position (Fig 1F). However, the intensity of bioluminescent signals from the same animal remained stable with the animal in the prone position when the exposure time and camera distance were fixed. The strongest bioluminescent signals were documented with the animal in the prone position, when the camera was above the bulla. We noted that other anatomic spaces in the head or neck (i.e., the larynx, pharynx, or sinuses) did not appear to become concomitantly infected.

Effects of Bullar Wall and Skin on Bioluminescent Signal Intensity. It is known that the chinchilla bulla, including the skin and bullar bone shell, is only 2 to $3 \mathrm{~mm}$ from the surface. To study the effect of the bullar wall and skin on bioluminescent signal detection, we scanned $0.2 \mathrm{~mL}$ of bacterial suspension both outside the bulla and inside the bulla of 10 chinchillas. The average pre-injection bioluminescent signal was $9.74 \times$ $10^{7}$ (photons per second, Fig 1G), whereas the average post-injection signal 
was $2.90 \times 10^{6}$ (photons per second, Fig $1 \mathrm{H}$ ). Thus, there was an average 34-fold

decrease in photons per second per square centimeter upon injection into an animal.
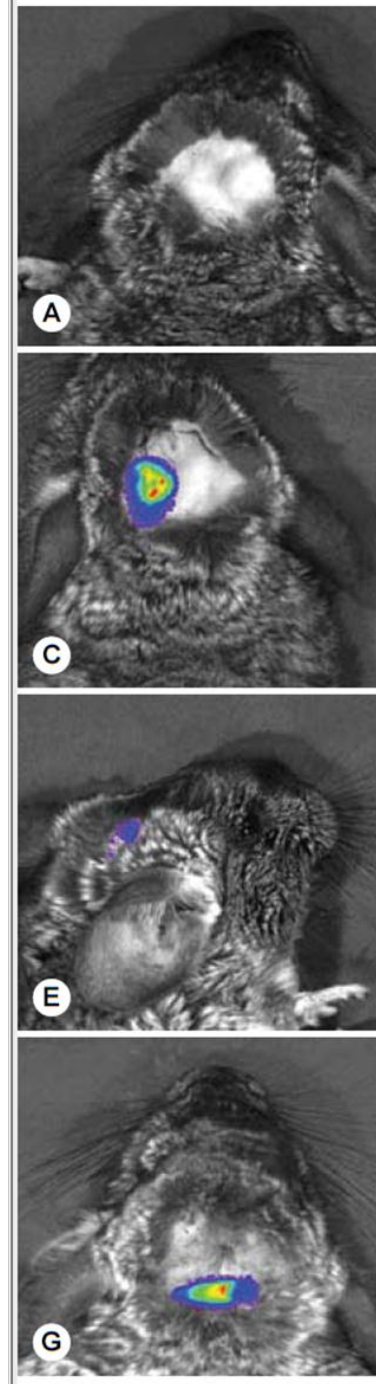
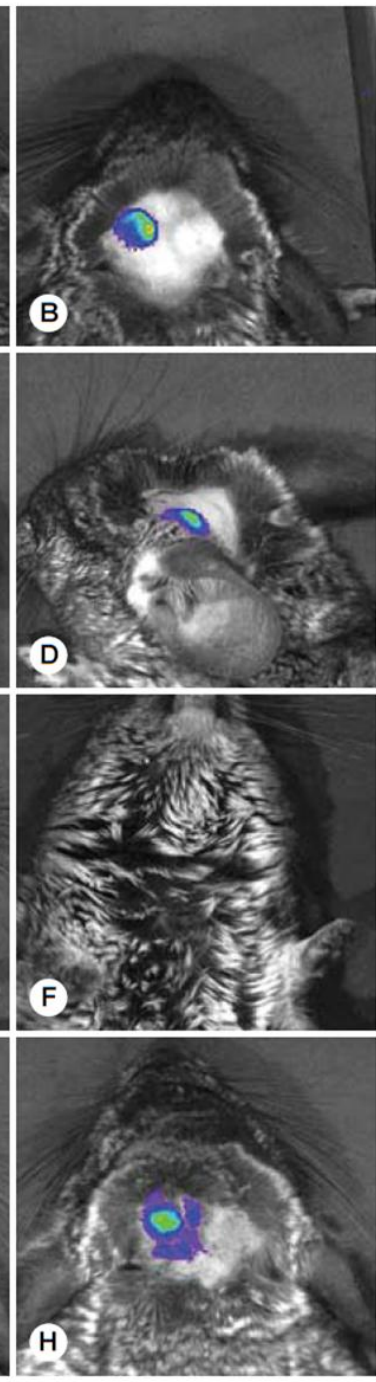
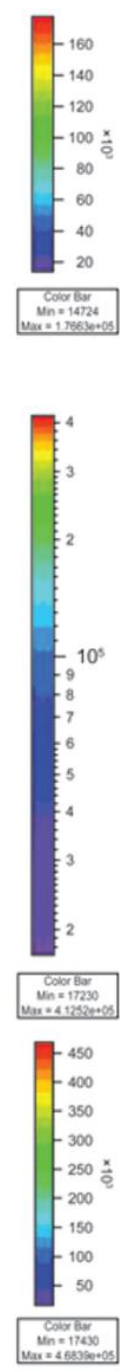

Fig 1. View of top of chinchilla head with animal in prone position. A) Post-inoculation image (hour 0). Fur overlying middle ear bullae of chinchilla has been removed to allow identification of left middle ear bulla for needle inoculation. B) Day 1 image (24 hours after inoculation). Color-coded region over left bulla represents bacterial growth in middle ear. C-E) Acute otitis media in one chinchilla with varied angles of exposure: C) prone, D) left lateral, E) right lateral, and F) supine. High signal intensity was observed when $0.2 \mathrm{~mL}$ of Xen 10 pneumococcus (approximately $10^{8}$ colonyforming units) was placed in transparent vial immediately outside bulla of anesthetized animal (G), whereas low signal intensity was noted when more than $0.2 \mathrm{~mL}$ of Xen 10 was injected into bulla approximately 5 minutes later (H). Note multi-compartment distribution pattern of bioluminescent bacteria in bulla. Image color scale units are photons per second per square centimeter.

Comparison of Bioluminescent Signal Intensities During AOM. In recognition of signal intensity stability above the bulla, we documented bioluminescent signals from chinchillas in the prone position with a fixed exposure parameter of the camera along the AOM path. All animals underwent imaging 24 hours after inoculation. Subsequently, we performed imaging regularly (typically every 48 hours) to assess for progression and 
resolution of disease. Representative bioluminescent imaging of the bulla from the beginning of infection on day 1 to the resolution of infection on day 12 is shown in Fig 2A, and its quantitative analysis is shown in Fig 2B.
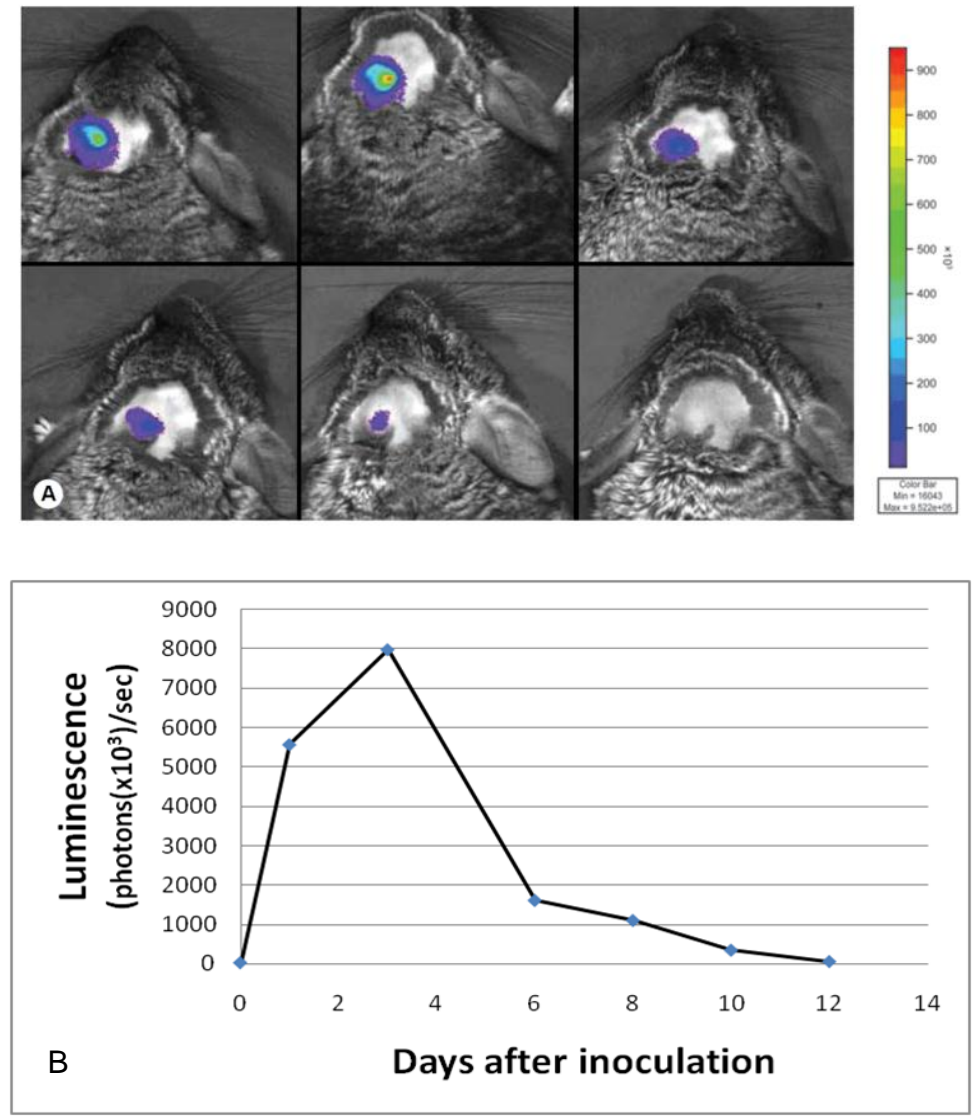

Fig 2. Time-dependent progression of acute otitis media in parallel with intensity changes of bioluminescent signals. A) Progressive series of bioluminescence images through acute phase of otitis media in chinchilla after injection of Xen 10 at 50 colony-forming units per bulla. Top row — days 1, 3, and 6; bottom row - days 8 , 10 , and 12. Image color scale units are photons per second per square centimeter. B) Quantitative analysis of bioluminescent signal intensity (photon counts) for associated images in A performed with Living Image software's regionof-interest function.

Spread of Bioluminescent Bacteria From Injected Bulla to Non-injected Bulla. During the study, no animals demonstrated bioluminescent signal from other areas (nose, sinuses, lungs, etc.). Interestingly, Fig 3 demonstrates that although AOM was the only manifestation of bacterial growth observed, a contralateral infection of AOM with bioluminescent pneumococcus could occur spontaneously. Presumably, this represents bacterial transport down the eustachian tube of the inoculated bulla and ascent through the contralateral eustachian tube causing a secondary AOM. 

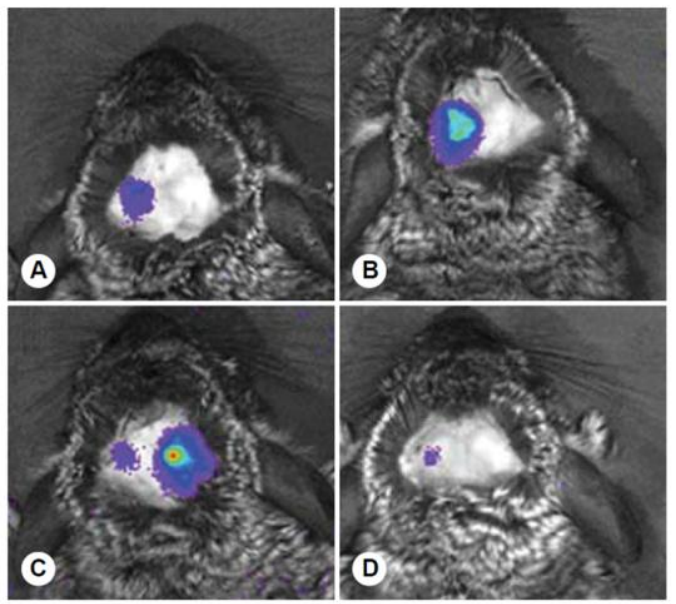

Fig 3. Dissemination of acute otitis media from one bulla to other of chinchilla, with time lag. Left bulla was injected with Streptococcus pneumoniae at 50 colony-forming units. A) Bioluminescent signals appeared in left ear on day 1 , and right ear was negative. B) Signals on left became strong, and peaked on day 3 , and right bulla remained negative. C) Signals weakened on day 5 , and non-injected bulla became infected. D) Signals had weakened on both left and right bullae on day 9 . Image color scale units are photons per second per square centimeter.

\section{Quantitative Analysis of Otitis Media With and Without Antibiotic Treatment in}

Chinchillas. Before we inoculated any animals with pneumococcus, we conducted an amoxicillin feasibility trial to ensure that amoxicillin caused no adverse effects in the animals. Amoxicillin therapy has been used for otitis media in chinchillas in many previous studies. ${ }^{18-20}$ Amoxicillin has been clearly shown to improve pneumococcusbased otitis media in this model $1{ }^{10}$ Amoxicillin, however, is also known to eradicate microbial flora in chinchillas, leading to diarrhea, dehydration, and sometimes death. Three chinchillas were provided a 10-day course of amoxicillin dosed twice daily by oral gavage. The 3 animals in this feasibility study were also anesthetized every other day with ketamine hydrochloride $(25 \mathrm{mg} / \mathrm{kg})$. None of the 3 animals showed adverse effects from either the amoxicillin or the ketamine.

The inoculated animals were submitted to imaging for middle ear infection between 22 and 26 hours after injection. Animals that showed no bioluminescent signals in the bulla and animals that died within the first 36 hours of infection were excluded. The animals were randomized to receive either oral amoxicillin ( $30 \mathrm{mg} / \mathrm{kg}$ per day) therapy with 
Bene-Bac probiotic gel or no antibiotic therapy. Baseline bioluminescent images were obtained on day 1 , and photon counts were quantified for each animal. The animals again underwent imaging on day 3 , after receiving 4 doses of amoxicillin, and photon counts were again obtained. For those animals that received antibiotics, the photon counts approached baseline levels. The controls were all noted to have increased photon counts when we compared the day 1 intensity (average, $1.5 \times 10^{6}$ photons per second per square centimeter) to the peak intensity on day 3 (average, $5.1 \times 10^{6}$ photons per second per square centimeter, a 2.4-fold increase in signal), consistent with the profile shown in Fig 2. Of those animals that received amoxicillin, a marked decrease in average signal intensity was noted between day $1\left(1.6 \times 10^{6}\right.$ photons per second per square centimeter $)$ and day $3\left(3.8 \times 10^{4}\right.$ photons per second per square centimeter). The difference in signal intensity between treated and untreated animals on day 3 ( 48 hours after initiation of therapy) was 24.11 -fold.

\section{DISCUSSION}

Bioluminescent imaging technology is powerful for tracing the infectious processes of human disease in animal models. The technology features noninvasive imaging of progressive disease processes in vivo and provides semi-quantitative information about bacterial loads in the living animal by exploiting photon-emitting enzymatic reactions in bacteria. This study demonstrates the power of using genetically engineered bioluminescent bacteria to gain new insights into the infectious process of bacteria and the response to antibiotics. It provides researchers with a new tool for assessing otitis 
media, evaluating the therapeutic efficacy of current antibiotics, and discovering potential therapeutic interventions for otitis media.

In the past, measures of bacterial growth within the middle ear were restricted to invasive measures, from repetitive needle aspiration to euthanizing animals at specifically timed points of the illness. Bioluminescent models such as this one eliminate those invasive and lethal practices from data collection. It is convenient to monitor the infectious process, inflammatory degree and scope, and potential disseminations and complications in the living animal by assessing bioluminescent signal intensity and location. Importantly, $S$ pneumoniae (Xen 10) is a strain that shows bioluminescent signals when growing, so it is easy to determine whether infection is active or inactive. This strain is also convenient for evaluating the efficacy of antibiotic therapeutic interventions in AOM. As expected, the scope and intensity of bioluminescent signals are related to the degree and scope of AOM in the same body position of an animal on different days. In other words, bioluminescent signals parallel the living bacterial metabolic activity in the middle ear cavity when a camera is at a fixed angle and distance.

The chinchilla model was chosen for this study because it has been shown to closely parallel the human continuum of otitis media, with an acute inflammatory phase that lasts between 1 and 2 weeks and is followed by an effusion phase that lasts 1 to 2 months. Further, common bacterial strains that cause AOM in humans have been shown to cause similar infections in chinchillas. There are no monitoring capabilities that are lost with 
this model; otomicroscopy, tympanometry, and histopathology are all still possible and can be used to support the bioluminescent data. The ability to quantify the severity of infection by photon counts allows the investigator to compare treatment arms with a precision that is unparalleled in otitis media research.

It is noted that chinchillas are susceptible to S pneumoniae (Xen 10). As in previous experiments with chinchillas, the animals in this study became markedly ill with the pneumococcal infection. This is a known and unfortunate consequence of using pneumococcus that contains surface protein $\mathrm{A}$ and surface antigen $\mathrm{A}$ in chinchillas. ${ }^{18}$ The model in chinchillas is similar to that in mice in terms of middle ear susceptibility to infection by S pneumonia and pathological changes following middle ear infection. ${ }^{21}$ There is likely ample opportunity to overcome this challenge, however, as many different serotypes and strains of bioluminescent otitis media pathogens are in development. In fact, a small series of commercially available bioluminescent pneumococcus strains are available from the suppliers of Xen 10. There are certain to be other, less virulent strains that provide bioluminescent infection equivalent to that of Xen 10 in the chinchilla model.

The challenge in directly correlating the bioluminescence levels to bacterial loads in this model stems from imaging capabilities and animal anatomy. The imaging system cannot detect trace amounts of bacteria within an animal. The bacterial bioluminescence detection threshold (approximately $10^{4} \mathrm{CFU} / \mathrm{mL}$ ) represents a bacterial concentration that 
is higher than the concentration that has been demonstrated to lead to disease (500 $\mathrm{CFU} / \mathrm{mL}$ ). In this study, bacterial collections in transparent vials with concentrations of less than $10^{4} \mathrm{CFU} / \mathrm{mL}$ could not be detected by bioluminescence. The level of $10^{4}$ $\mathrm{CFU} / \mathrm{mL}$ appeared to represent the baseline detection threshold achievable with this generation of technology and this strain of bacteria. This limitation is likely threefold: 1) the baseline amount of photons produced in animals by natural metabolic processes, as well as by inanimate objects such as the plastic containers and adhesive tapes used to isolate the animal in the imaging system; 2) the imaging device's innate detection threshold; and 3) these bacteria's inherent bioluminescence level. Bacterial concentrations that were below the threshold of detection in this model were never shown to concurrently produce middle ear effusions or observable symptoms. Nonetheless, small bacterial populations, below the detection thresholds, were shown in this study to blossom into robust infections. Thus, while this technology is quite powerful, it cannot detect small, subclinical concentrations of live bacteria.

An additional challenge in quantifying bacterial count by bioluminescence is the multicompartmental nature of the chinchilla bulla. These compartments are divided into superior and inferior divisions with smaller accessory chambers. ${ }^{22,23}$ The total mean volume of the bulla compartments is just over $2 \mathrm{~mL}$ in an adult chinchilla. ${ }^{24}$ As we demonstrated in this study, the overlying skin and bone on the bulla may reduce the bioluminescent photon counts by 1 to 2 orders of magnitude. The chambers are known to communicate freely with each other. Consequently, bioluminescent signals may differ 
within the same animal, depending upon whether the bacteria occupy either of the individual chambers or occupy both simultaneously. Further, as this study shows, the angle of imaging can have a considerable impact on the device's ability to detect the photon signal.

As reported in previous articles, the thickness of tissues between the metabolically active bacteria and the imager reduces the concentration of photons captured by the imager. Figure 1C-F supports this concept, as the "lateral" images show a reduced bioluminescent signal and the "supine" image shows none. Many factors contribute to reduced photon sensitivity when bioluminescent cells are viewed in vivo, including the number of bioluminescent cells, the efficiency of the gene promoter and cellular metabolism, the tissue depth, and the signal impedance caused by overlying soft tissue pigmentation and fur. ${ }^{11,14,15}$ Contag et $a^{25}$ estimated a roughly 10 -fold loss of photon sensitivity for each centimeter of overlying soft tissue. Our 34-fold difference appears larger than that estimate. Although the chinchilla bulla is only 2 to $3 \mathrm{~mm}$ from the surface, it is encased in mineralized bone, which likely absorbs many photons and accounts for the majority of lost signal. This animal model of bioluminescence technology in AOM offers new strengths and insights into the pathogenesis of the illness. However, although bioluminescence technology offers much more powerful monitoring capabilities than were possible previously, it still does not eliminate the animal-to-animal variability inherent in virtually all animal studies. 


\section{CONCLUSIONS}

Chinchilla-based models have remained a standard approach to studying otitis media over the past few decades. Bioluminescent microbes offer a unique opportunity to reinvent this model to study the infectious process of otitis media in finer detail than ever before and offer a new platform upon which to evaluate future treatment options. With this technology, we are moving into a new era of direct visualization of the infectious process of middle ear pathogens and objective evaluation of the relevant medical therapy. 


\section{REFERENCES}

1. Freid VM, Makuc DM, Rooks RN. Ambulatory health care visits by children: principal diagnosis and place of visit. Vital Health Stat 131998 May;(137):1-23.

2. Teele DW, Klein JO, Rosner B. Epidemiology of otitis media during the first seven years of life in children in greater Boston: a prospective, cohort study. J Infect Dis 1989; 160:83-94.

3. Gates GA. Cost-effectiveness considerations in otitis media treatment. Otolaryngol Head Neck Surg 1996;114:525-30.

4. Bluestone CD. Otitis media in children: to treat or not to treat? N Engl J Med 1982;306:1399-404.

5. Dagan R. Treatment of acute otitis media - challenges in the era of antibiotic resistance. Vaccine 2000;19(suppl 1):S9-S16.

6. Giebink GS. The prevention of pneumococcal disease in children. N Engl J Med 2001;345:1177-83.

7. Daly KA, Hunter LL, Giebink GS. Chronic otitis media with effusion. Pediatr Rev 1999;20:85-94.

8. Eskola J, Kilpi T, Palmu A, et al; Finnish Otitis Media Study Group. Efficacy of a pneumococcal conjugate vaccine against acute otitis media. N Engl J Med 2001;344:403-9.

9. Juhn SK, Tolan CJ, Antonelli PJ, Giebink GS, Goycoolea MV. The significance of experimental animal studies in otitis media. Otolaryngol Clin North Am 1991;24:81327.

10. G iebink GS. Otitis media: the chinchilla model. Microb Drug Resist 1999;5:57-72.

11. Sato A, Klaunberg B, Tolwani R. In vivo bioluminescence imaging. Comp Med 2004;54:631-4.

12. Bakaletz LO, Ohashi Y, Demaria TF, Lim DJ. Effect of formalin-fixed Hemophilus influenzae and Streptococcus pneumonia on dye transport by the chinchilla eustachian tube. Acta Otolaryngol 1989;107:235-43.

13. Cantekin EI. Bacterial DNA fragments in otitis media with effusion [Comment]. JAMA 1996;275:186.

14. Sadikot RT, Blackwell TS. Bioluminescence: imaging modality for in vitro and in vivo gene expression. Methods Mol Biol 2008;477:383-94.

15. Zinn KR, Chaudhuri TR, Szafran AA, et al. Noninvasive bioluminescence imaging in small animals. ILAR J 2008;49: 103-15.

16. Francis KP, Yu J, Bellinger-Kawahara C, et al. Visualizing pneumococcal infections in the lungs of live mice using bioluminescent Streptococcus pneumoniae transformed with a novel gram-positive lux transposon. Infect Immun 2001;69:33508.

17. Hausdorff WP, Yothers G, Dagan R, et al. Multinational study of pneumococcal serotypes causing acute otitis media in children. Pediatr Infect Dis J 2002;21:100816. 
18. Huang Y, Ji P, Inano A, Yang Z, Giebink GS, Sawchak RJ. Microdialysis studies of the middle ear distribution kinetics of amoxicillin in the awake chinchilla. J Pharm Sci 2001; 90:2088-98.

19. Sawchuk RJ, Cheung BW, Ji P, Cartier LL. Microdialysis studies of the distribution of antibiotics into chinchilla middle ear fluid. Pharmacotherapy 2005;25:140S-145S.

20. Huang Y, Yang Z, Cartier L, Cheung B, Sawchuk RJ. Estimating amoxicillin influx/efflux in chinchilla middle ear fluid and simultaneous measurement of antibacterial effect. Antimicrob Agents Chemother 2007;51:4336-41.

21. Melhus A, Ryan AF. A mouse model for acute otitis media. APMIS 2003;111:98994.

22. Hanamure Y, Lim DJ. Anatomy of the chinchilla bulla and eustachian tube: I. Gross and microscopic study. Am J Otolaryngol 1987;8:127-43.

23. Daniel HJ III, Fulghum RS, Brinn JE, Barrett KA. Comparative anatomy of eustachian tube and middle ear cavity in animal models for otitis media. Ann Otol Rhinol Laryngol 1982;91:82-9.

24. Jossart GH, Erdmann GR, Levitt DG, et al. An experimental model for measuring middle ear antimicrobial drug penetration in otitis media. Pharm Res 1990;7:1242-7.

25. Contag CH, Contag PR, Mullins JI, Spilman SD, Stevenson DK, Benaron DA. Photonic detection of bacterial pathogens in living hosts. Mol Microbiol 1995;18:593-603. 